\title{
Preferência pelo tipo de parto, fatores associados à expectativa e satisfação com o parto
}

Ana Carolina Lima Silva ${ }^{1}$, Hévyllin Cipriano Rodrigues Félix², Maria Beatriz Guimarães Ferreira ${ }^{3}$, Anneliese Domingues Wysocki ${ }^{4}$, Divanice Contim ${ }^{5}$, Mariana Torreglosa Ruiz ${ }^{6}$

\footnotetext{
${ }^{1}$ Enfermeira. Uberaba, MG, Brasil. E-mail: aninhacarol.enf@gmail.com.

${ }^{2}$ Discente do curso de Graduação da Universidade Federal do Triângulo Mineiro. Uberaba, MG, Brasil. E-mail:

hevyllin_rodrigues@hotmail.com.

${ }^{3}$ Enfermeira, Doutora em Enfermagem Fundamental. Professor da Universidade de Uberaba. Uberaba, MG, Brasil. E-mail: mariabgfo@gmail.com.

${ }^{4}$ Enfermeira, Doutora em Ciências da Saúde. Professora Adjunto da Universidade Federal de Mato Grosso do Sul. Três Lagoas, MS, Brasil. E-mail:

lilisew@yahoo.com.br.

${ }^{5}$ Enfermeira, Doutora em Enfermagem. Professora Adjunto da Universidade Federal do Triângulo Mineiro. Uberaba, MG, Brasil. E-mail: d.contim@uol.com.br.

${ }^{6}$ Enfermeira, Doutora em Enfermagem em Saúde Pública. Professora Adjunto da Universidade Federal do Triângulo Mineiro. Uberaba, MG, Brasil. E-mail:

marianatorreglosa@hotmail.com.
}

Recebido: 15/11/2016.

Aceito: 01/06/2017.

Publicado: 01/09/2017.

Como citar esse artigo:

Silva ACL, Félix HCR, Ferreira MBG, Wysocki $A D$, Contim D, Ruiz MT. Preferência pelo tipo de parto, fatores associados à expectativa e satisfação com o parto. Rev. Eletr. Enf. [Internet]. 2017 [acesso em: ____ ] 19:a34. Disponível em: http://dx.doi.org/10.5216/ree.v19.44139.

\section{RESUMO}

Este estudo teve por objetivo analisar a preferência e a satisfação das mulheres com o tipo de parto e a associação com as características sociodemográficas e obstétricas. Estudo transversal, realizado em três etapas, com amostra de 190 puérperas. Os resultados mostraram que $68,9 \%$ desejavam parto normal e 31,1\% cesárea. Foram associadas à preferência pelo parto normal: primeira gestação $(p=0,042)$ e experiência anterior de parto normal $(p<0,001)$; entre as mulheres que tiveram cesárea prévia a preferência prevaleceu por esta $(p<0,001)$. Não foram evidenciadas diferenças estatísticas associadas a outras variáveis, bem como, a satisfação das puérperas. A regressão logística binária indicou associação entre experiência prévia e expectativa por tipo de parto. A experiência com parto anterior influenciou na preferência pelo tipo de parto, entretanto, não houve diferenças na satisfação das puérperas, independente se o desfecho correspondeu a expectativa.

Descritores: Cesárea; Parto Normal; Satisfação Pessoal; Enfermagem

\section{INTRODUÇÃO}

A indicação do tipo de parto deve ser baseada em motivos clínicos consistentes e em situações específicas. A cesárea é um procedimento cirúrgico e tem como finalidade intervir quando riscos são maiores diante dos benefícios do parto normal, devendo ser indicada, apenas em casos necessários ${ }^{(1)}$. Observa-se elevação expressiva das taxas de cesáreas em praticamente todo o mundo, ultrapassando o percentual de $10 \%$ a $15 \%$ dos partos, que é considerado aceitável e justificável pela Organização Mundial de Saúde (OMS) $)^{(1)}$.

No Brasil, atualmente a cesárea é o modo mais comum de nascimento, representando $56 \%$ dos partos, 
índice este que contribuiu para a criação de protocolo com diretrizes para cesáreas, que tem por objetivo reduzir esses números, uma vez que a indicação inadequada pode favorecer a morbimortalidade materna e infantil ${ }^{(2)}$. Estudo de base nacional intitulado 'Nascer no Brasil' apontou taxa de 51,9\% de cesáreas, e destas, 45,5\% ocorreram em gestações de baixo risco, $80 \%$ foram indicadas devido ao primeiro parto ter sido cesárea e, $88 \%$ das mulheres sequer entraram em trabalho de parto ${ }^{(3)}$.

De acordo com o American College of Obstetricians and Gynecologists, a cesárea eletiva indicada antes de 40 semanas gestacionais, não permite o completo desenvolvimento cerebral do feto, que tem seu término estimado entre 35 a 39 semanas. Diante disso, o neonato pode apresentar problemas respiratórios, dificuldades de controle térmico, dificuldades de sucção/alimentação, icterícia por excesso de bilirrubina, problemas auditivos, visuais, de aprendizagem e de conduta. Além disto, o neonato apresenta maior risco de: morte, necessidade de internação em Centros de Terapia Intensiva, período prolongado de internação e maior procura por serviços de saúde no primeiro ano de vida. Além dos riscos inerentes ao procedimento cirúrgico, as mulheres submetidas à cesárea apresentam risco de desenvolvimento de infecções e hemorragias, e, em gestações futuras, podem apresentar maior risco para placenta prévia, acretismo placentário, hemorragia pós-parto e histerectomia. Desta forma, preconiza-se que na ausência de indicação materna ou fetal, o parto vaginal é sempre o mais seguro e apropriado ${ }^{(4)}$.

Assim, dentre as indicações de cesáreas são justificáveis: distócias ou falha na progressão do trabalho de parto após instalação do mesmo, desproporção cefalopélvica, intervalo interpartal menor que dois anos após cesárea, apresentações anômalas fetais, sofrimento fetal agudo, mecônio espesso (sofrimento fetal) e alterações na frequência cardíaca fetal ${ }^{(5)}$.

Após uma cesárea prévia, riscos devem sempre ser avaliados por meio de escores objetivos para indicar a próxima cesárea, uma vez que o parto vaginal pode ser possível e não está contraindicado nestes casos. Apenas após duas cesáreas ou intervalo interpartal inferior a dois anos, indica-se a cirurgia ${ }^{(6)}$.

Apesar de aumentar o risco de morbimortalidade tanto para mãe quanto para o recém-nascido, muitas gestantes desejam a cesárea como via de parto. O medo está entre os motivos que podem influenciar a expectativa e a escolha da gestante, este é um dos agentes estressantes mais relatados por diferentes motivos: medo da dor, medo de não conseguir ter o parto, medo da forma como será conduzido pelos profissionais, sendo as principais justificativas para a preferência pela cesárea, pois é vista por muitas como indolor, rápida e segura ${ }^{(7-8)}$.

Vale destacar que a informação sobre tipos de parto, riscos e benefícios são direitos universais das mulheres grávidas previstos pela Aliança Global para Maternidade Segura, e a preferência e escolha da mulher pelo tipo de parto deve ser considerada sempre em posse deste conhecimento ${ }^{(9)}$.

Diante da realidade de altos índices de cesárea no Brasil e suas possíveis complicações, justifica-se a importância de identificar preferências, indicações e desfechos de mulheres submetidas à cesárea, com vistas a elucidar a temática e analisar como a assistência tem sido prestada, possibilitando traçar estratégias para favorecer a qualidade da assistência e segurança do binômio mãe-filho. 
Assim, este estudo teve por objetivo: analisar a preferência e a satisfação das mulheres com o tipo de parto e a associação com as características sociodemográficas e obstétricas.

\section{MÉTODO}

Trata-se de um estudo quantitativo transversal realizado nas dependências do Hospital de Clínicas da Universidade Federal do Triângulo Mineiro (HCUFTM).

A pesquisa foi aprovada pelo Comitê de Ética em Pesquisa da Universidade Federal do Triângulo Mineiro sob parecer $n^{\circ} 862.636$ de 24 de outubro de 2014 , atendendo às orientações inerentes ao protocolo de pesquisa contido na Resolução n 466/2012 do Conselho Nacional de Saúde. Todas as participantes após esclarecimentos assinaram o Termo de Consentimento Livre e Esclarecido (TCLE), e as com idade inferior a 18 anos, tiveram o consentimento dos seus responsáveis legais associado ao seu assentimento.

A coleta de dados foi realizada em três etapas: a primeira para identificar a preferência pelo tipo de parto entre gestantes, a segunda, após a ocorrência do parto, para analisar o desfecho entre as puérperas e a terceira que buscou avaliar a satisfação das mesmas com o parto.

Participaram da primeira etapa da pesquisa gestantes de risco habitual e de alto risco, hemodinamicamente estáveis e em boas condições clínicas, a partir da 30ạ semana gestacional atendidas no ambulatório do hospital (HCUFTM). Gestantes com malformações fetais incompatíveis com a vida (detectadas por avaliação e diagnósticos médicos); que apresentavam déficit cognitivo e/ou que tiveram como desfecho aborto, óbito fetal ou natimorto e aquelas que tiveram os partos realizados em outras instituições ou municípios foram excluídas.

A amostra foi não probabilística com coleta de dados por conveniência, onde foram incluídas 211 gestantes, no período de dezembro de 2014 a fevereiro de 2015. Entre a primeira e a segunda etapa foram excluídas 21 participantes, pois não tiveram o parto na instituição. As perdas foram retiradas da análise do estudo, de modo que a amostra final foi constituída apenas pelas participantes das duas etapas do estudo, que totalizaram 190 participantes.

Na primeira etapa foram realizadas entrevistas por meio da aplicação de instrumento estruturado construído pelos próprios autores e submetido a estudo-piloto com 10 gestantes, que foram excluídas da análise.

O instrumento contemplava as seguintes variáveis: informações sociodemográficas (idade; procedência, cor autorreferida, companheiro, ocupação e escolaridade) condições de saúde (antecedentes familiares, tabagismo, etilismo, uso de drogas ilícitas e patologias), história obstétrica atual e pregressa (número de gestações, partos prévios, abortos, número de consultas pré-natais, idade gestacional no momento da entrevista). Após este bloco de questões, foram feitas duas questões, sendo, uma fechada que questionava qual o tipo de parto desejado e uma aberta, em que a participante justificava o motivo pela preferência. Destaca-se que em caso de informação ignorada, a mesma foi retirada da análise estatística. Adicionalmente às entrevistas foram coletadas informações do cartão de pré-natal e dos prontuários das 
mesmas. As gestantes foram abordadas durante espera ou após consulta, orientadas sobre os objetivos e convidadas a participar. A entrevista ocorreu em consultório, respeitando princípios de sigilo e privacidade.

Para realização da segunda etapa, os pesquisadores optaram pela lista de pacientes internadas na Enfermaria de Ginecologia e Obstetrícia do hospital, o registro das mulheres que participaram da primeira etapa, tendo como base o cálculo da data provável do parto ou o agendamento do parto. Nesta etapa foi coletada informação sobre o tipo de parto realizado e justificativa, em caso de cesárea. Estas informações foram coletadas nos prontuários e, caso não encontradas as puérperas durante a coleta, foram colhidas informações junto ao setor de arquivo médico do hospital.

A terceira etapa do estudo foi realizada no intervalo de sete a 15 dias após alta. As mulheres foram contactadas por telefone para opinar a respeito da satisfação com o parto, respondendo apenas se estavam satisfeitas ou não com a experiência e se insatisfeitas, qual(is) o(s) motivo(s). Optou-se por este método, para evitar viés de resposta, uma vez que as mesmas estariam alocadas nas enfermarias da instituição durante a segunda etapa. Foram realizadas três tentativas de contato a partir do número fornecido, entretanto, não foi possível contato com 19 puérperas, sendo questionadas sobre satisfação 171 puérperas.

Para a análise, adotou-se a técnica de dupla digitação com posterior validação, empregando-se o aplicativo Microsoft Excel ${ }^{\circledR}$. Utilizou-se na análise estatística o aplicativo Statistical Package for the Social Sciences ${ }^{\circledR}$ versão 20.0. Inicialmente realizou-se a análise descritiva dos dados relativos às variáveis sociodemográficas e obstétricas. A associação entre preferência por tipo de parto e variáveis de interesse foi verificada pelo teste estatístico qui-quadrado. Para verificar a magnitude das associações, utilizaram-se razões de prevalência (RP), razões de chances (RS) e seus respectivos intervalos de confiança (95\%). Para ratificar associações realizou-se a regressão logística binária. 0 nível de significância adotado foi de 0,05.

\section{RESULTADOS}

A idade média das mulheres foi de $26,2 \pm 6,4$, variando de 14 a 41 anos. Destas, $6,3 \%$ eram adolescentes e 10,9 \% tinham idade igual ou superior a 35 anos; a maioria possuía companheiro $(83,2 \%)$ e não exercia atividade remunerada $(54,2 \%)$.

Quanto às condições clínicas, antecedentes familiares e hábitos, 26,3\% não possuíam antecedentes familiares patológicos e 16,3\% possuíam antecedente familiar de hipertensão arterial; 6,3\% consumiam bebida alcoólica; 7,4\% eram tabagistas e apenas uma mulher declarou fazer uso de drogas ilícitas. Quando questionadas sobre doenças prévias ou em curso, $24,2 \%$ mencionaram pelo menos uma doença, sendo mais frequentes síndromes hipertensivas (21,3\%), hipotireoidismo $(12,8 \%)$ e epilepsia $(8,5 \%)$.

Em relação à história obstétrica, o número de gestações variou de uma a nove, com média de 2,56 $( \pm 1,5)$, mais frequentes secundigestas $(35,8 \%)$; número médio de filhos vivos de $1,18( \pm 1,3)$, variando de zero a seis e, $24,7 \%$ tinham tido aborto anterior como desfecho de gestação prévia. A idade gestacional no momento da entrevista variou de 30 a 40 semanas, com média de $33,8( \pm 3,4)$ semanas gestacionais.

Quando questionadas sobre expectativa em relação ao tipo de parto, 68,9\% desejava parto normal, 
destas, 55,7\% acreditava que este parto proporcionaria recuperação materna mais rápida; 14,5\% citou que era o parto mais natural e saudável para mãe e recém-nascido; 8,4\% citou a dor no parto normal como momentânea e 8,4\% queria ter novamente a experiência do parto normal, uma vez que a anterior foi bem sucedida.

Entre mulheres que tinham expectativa pela cesárea, 30,5\% referiu a iteratividade (mais de duas cesáreas) como causa; 23,7\% atribuiu ao medo da dor e, 15,3\% a segurança da cirurgia.

Dados do prontuário apontaram que 50,9\% dos partos se ultimaram por cesárea; 48,1\% por parto normal e 0,9\% por parto vaginal instrumental (fórceps). Nas justificativas da cesárea presentes no prontuário das puérperas, $18,9 \%$ foram classificadas como emergenciais e entre os motivos de indicação foram mais frequentes: iteratividade $(23,2 \%)$, sofrimento fetal $(16,8 \%)$; macrossomia $(10,5 \%)$, falha de indução $(10,5 \%)$ e complicações maternas $(10,5 \%)$.

$\mathrm{Na}$ análise entre as características sociodemográficas com a preferência pelo tipo de parto normal evidenciou que não houve associação (Tabela 1).

Tabela 1: Associação da preferência por tipo de parto normal com as características sociodemográficas. Uberaba, MG, Brasil, 2015.

\begin{tabular}{|c|c|c|c|c|c|c|c|c|c|}
\hline \multirow{2}{*}{ Variável } & \multicolumn{2}{|c|}{ Normal } & \multicolumn{2}{|c|}{ Cesárea } & \multicolumn{2}{|c|}{ Total } & \multirow{2}{*}{$\mathbf{R P}$} & \multirow{2}{*}{ RS } & \multirow{2}{*}{ p } \\
\hline & $\mathbf{n}$ & $\%$ & $n$ & $\%$ & $\mathbf{n}$ & $\%$ & & & \\
\hline \multicolumn{10}{|l|}{ Idade $(n=190)$} \\
\hline$<26$ & 70 & 72,9 & 26 & 27,1 & 96 & 50,5 & 1,12 & 1,46 & 0,232 \\
\hline$>26$ & 61 & 64,9 & 33 & 35,1 & 94 & 49,5 & $(0,93-1,36)$ & $(0,78-2,70)$ & \\
\hline \multicolumn{10}{|l|}{ Atividades ocupacionais $(n=188)$} \\
\hline Sem remuneração & 72 & 66,1 & 37 & 33,9 & 109 & 58,0 & 0,92 & 0,75 & 0,374 \\
\hline Atividades remuneradas & 57 & 72,2 & 22 & 27,8 & 79 & 42,0 & $(0,76-1,11)$ & $0,40-1,41)$ & \\
\hline \multicolumn{10}{|l|}{ Escolaridade $(n=190)$} \\
\hline Sem escolaridade até ensino fundamental & 84 & 67,7 & 40 & 32,3 & 124 & 65,3 & 0,95 & 0,85 & 0,623 \\
\hline Ensino médio ou superior & 47 & 71,2 & 19 & 28,8 & 66 & 34,7 & $(0,78-1,16)$ & $(0,44-1,63)$ & \\
\hline \multicolumn{10}{|l|}{ Companheiro $(n=190)$} \\
\hline Possui companheiro & 108 & 68,4 & 50 & 31,6 & 158 & 83,2 & 0,95 & 0,85 & 0,695 \\
\hline Não possui companheiro & 23 & 71,9 & 9 & 28,1 & 32 & 16,8 & $(0,75-1,21)$ & $(0,37-1,96)$ & \\
\hline \multicolumn{10}{|l|}{ Cor autorreferida $(n=190)$} \\
\hline Branca & 40 & 69,0 & 18 & 31,0 & 58 & 30,5 & 1,00 & 1,00 & 0,997 \\
\hline Não branca & 91 & 68,9 & 41 & 31,1 & 132 & 69,5 & $(0,41-1,20)$ & $(0,51-1,95)$ & \\
\hline \multicolumn{10}{|l|}{ Renda $(n=190)$} \\
\hline < 1 salário mínimo & 105 & 67,3 & 51 & 32,7 & 156 & 82,1 & 0,89 & 0,63 & 0,295 \\
\hline > 1 salário mínimo & 26 & 76,5 & 8 & 23,5 & 34 & 17,9 & $(0,71-1,09)$ & $(0,27-1,50)$ & \\
\hline
\end{tabular}

$\mathrm{Na}$ análise entre as características obstétricas com a preferência pelo tipo de parto normal evidenciou que houve associação com a primeira gestação $(p=0,042)$ e a experiência anterior de parto normal $(p<0,001)$; e entre as mulheres que tiveram cesárea prévia, houve maior preferência por nova cesárea $(p<0,001)$ (Tabela 2).

No modelo de regressão logística binária, a variável experiência anterior de parto normal permaneceu associada a esta via e a experiência de cesárea prévia associada à preferência por nova cesárea (Tabela 3), indicando que a experiência prévia influencia na expectativa. 
Tabela 2: Associação da preferência por tipo de parto normal com variáveis obstétricas. Uberaba, MG, Brasil, 2015.

\begin{tabular}{|c|c|c|c|c|c|c|c|}
\hline \multirow{2}{*}{ Variável } & \multicolumn{2}{|c|}{ Normal } & \multicolumn{2}{|c|}{ Cesárea } & \multirow{2}{*}{$\mathbf{R P}$} & \multirow{2}{*}{ RS } & \multirow{2}{*}{$\mathbf{p}$} \\
\hline & $\mathbf{N}$ & $\%$ & $\mathbf{n}$ & $\%$ & & & \\
\hline \multicolumn{8}{|l|}{ Consultas Pré-Natal $(n=190)$} \\
\hline$\geq 6$ & 84 & 70,0 & 36 & 30,0 & 1,04 & 1,14 & \multirow{2}{*}{0,681} \\
\hline$<6$ & 47 & 67,1 & 23 & 32,9 & $(0,85-1,28)$ & $(0,61-2,15)$ & \\
\hline \multicolumn{8}{|l|}{ Número de gestações $(n=190)$} \\
\hline Uma gestação & 38 & 80,9 & 9 & 19,1 & 1,24 & 2,27 & \multirow{2}{*}{0,042} \\
\hline Duas ou mais gestações & 93 & 65,0 & 50 & 35,0 & $(1,03-1,50)$ & $(1,02-5,07)$ & \\
\hline \multicolumn{8}{|l|}{ Parto normal prévio $(n=190)$} \\
\hline Experiência prévia & 62 & 83,8 & 12 & 16,2 & 1,41 & 3,52 & \multirow{2}{*}{$<0,001$} \\
\hline Sem experiência & 69 & 59,5 & 47 & 40,5 & $(1,18-1,69)$ & $(1,71-7,24)$ & \\
\hline \multicolumn{8}{|l|}{ Cesárea prévia ( $n=190)$} \\
\hline Experiência prévia & 24 & 35,8 & 43 & 64,2 & 0,41 & 0,08 & \multirow{2}{*}{$<0,001$} \\
\hline Sem experiência & 107 & 87,0 & 16 & 13,0 & $(0,30-0,57)$ & $(0,40-0,17)$ & \\
\hline \multicolumn{8}{|c|}{ Orientações sobre tipos de parto na gestação $(n=187)$} \\
\hline Recebeu orientações & 53 & 63,1 & 31 & 36,9 & 0,87 & 0,64 & \multirow{2}{*}{0,155} \\
\hline Não recebeu orientações & 75 & 72,8 & 28 & 27,2 & $(0,71-1,06)$ & $(0,34-1,19)$ & \\
\hline
\end{tabular}

Tabela 3: Modelo de regressão logística binária entre o desfecho expectativa por parto vaginal associado a variáveis obstétricas

\begin{tabular}{|c|c|c|c|c|}
\hline Variável & $\bar{n}$ & $\%$ & RCP & $p$ \\
\hline \multicolumn{5}{|l|}{ Número de gestações } \\
\hline Uma gestação & 67 & 35,3 & 1,236 & \multirow{2}{*}{0,729} \\
\hline Duas ou mais gestações & 123 & 64,7 & $(0,37-4,10)$ & \\
\hline \multicolumn{5}{|l|}{ Parto normal prévio } \\
\hline Experiência prévia & 74 & 38,9 & 0,388 & \multirow{2}{*}{0,044} \\
\hline Sem experiência & 116 & 61,1 & $(0,16-0,97)$ & \\
\hline \multicolumn{5}{|l|}{ Cesárea prévia } \\
\hline Experiência prévia & 47 & 24,7 & 12,113 & \multirow{2}{*}{$<0,001$} \\
\hline Sem experiência & 143 & 75,3 & $(4,56-32,16)$ & \\
\hline
\end{tabular}

Entre as 171 puérperas contactadas, não houve diferenças significativas $(p=0,407)$ na satisfação com o tipo de parto, independente se o desfecho correspondeu a expectativa (Tabela 4).

Tabela 4. Satisfação das puérperas com o tipo de parto e correspondência entre desfecho e expectativa ( $n=171)$. Uberaba, MG, Brasil, 2015.

\begin{tabular}{|c|c|c|c|c|c|c|c|}
\hline \multirow{2}{*}{ Desfecho e expectativa } & \multicolumn{2}{|c|}{ Satisfeita } & \multicolumn{2}{|c|}{ Não satisfeita } & \multirow{2}{*}{$\mathbf{R P}$} & \multirow{2}{*}{ RS } & \multirow{2}{*}{$\mathbf{p}$} \\
\hline & $\mathbf{n}$ & $\%$ & $\bar{n}$ & $\%$ & & & \\
\hline Sim & 111 & 94,1 & 7 & 5,9 & 1,04 & 1,66 & \multirow{2}{*}{0,407} \\
\hline Não & 48 & 90,6 & 5 & 9,4 & $(0,94-1,15)$ & $(0,50-5,47)$ & \\
\hline
\end{tabular}

\section{DISCUSSÃO}

O presente estudo apontou que a maioria das gestantes entrevistadas relatou preferência pelo parto normal. Semelhantemente, outros estudos brasileiros corroboram com esta preferência, com frequências variando de $58 \%$ a $80 \%{ }^{(10-11)}$.

A decisão pelo tipo de parto gera dúvidas, insegurança e medo. Mulheres que vivenciaram o parto normal possuem percepções positivas, sendo este mais tranquilo, rápido, simples e prático. A experiência anterior exerce papel fundamental na decisão, assim, mulheres que tiveram parto normal continuam optando pelo mesmo. Já primíparas, que possuem dúvidas acabam sendo influenciadas pela mídia, familiares 
e por quem já vivenciou o parto ${ }^{(9)}$.

Os motivos que levaram as participantes a preferirem o parto normal são reforçados por outros estudos que também encontraram a recuperação materna mais rápida, o parto mais natural e saudável para mãe e recém-nascido e a dor momentânea no parto ${ }^{(8,10-11)}$, além desses motivos os autores apontam: procedimentos mais rápidos, maior facilidade no aleitamento e experiência anterior de parto normal ${ }^{(8,10-11)}$.

Menos da metade das gestantes entrevistadas relataram expectativa pela cesárea, semelhante a outros estudos brasileiros que o percentual de preferência pela cesárea variou de 20 a $42 \%{ }^{(10-11)}$. Investigação realizada acerca dos aspectos relacionados à preferência pela cesárea concluiu que as motivações são o medo da dor do parto vaginal, insegurança na assistência local, experiência negativa no parto normal, desejar a realização de laqueadura e experiência positiva prévia ${ }^{(10-11)}$.

Contraditoriamente, um estudo apontou que $60 \%$ das gestantes relatavam preferência pela cesárea durante a gestação e a taxa de cesárea na amostra foi de $59,2 \%$. Quando analisados fatores associados à cesárea, observou-se maior ocorrência de cesáreas quando o mesmo médico que fez o atendimento prénatal assistiu a mulher no parto $(92,3 \%, p<0,001)$; idade materna superior a 30 anos de idade $(69,6 \%$, $p=0,005)$, com maior nível de escolaridade $(63,4 \%, p=0,004)$, gestações planejadas $(67,7 \%, p=0,003)$ e com história de cesárea prévia $(63,6 \%, p<0,001)$. Das cesáreas realizadas, $80 \%$ das mulheres sequer entraram em trabalho de parto e, $70 \%$ foram assistidas pelo mesmo médico no pré-natal e no parto, pelo sistema de saúde suplementar - atendimento por convênio $(p<0,001)$ e particular $(p<0,001)$, sendo a ocorrência de cesárea significante nestes $\operatorname{casos}^{(12)}$.

Embora a maioria das gestantes tenha desejado o parto normal, a maioria dos nascimentos ocorreu por cesárea. Estudo realizado com 81 gestantes no Rio Grande do Sul confirmam os alarmantes índices de cesárea no Brasil, o qual evidenciou que a preferência pelo tipo de parto pelas gestantes não interferiu no parto realizado, pois a maioria desejava o parto normal (75\%), porém a taxa de cesárea no município estudado apresenta índice de $89 \%^{(13)}$.

Estudo realizado em um hospital público de Picos (PI) apontou taxa semelhante de cesáreas (54,5\%), conforme os resultados do presente estudo ${ }^{(14)}$. Um estudo chinês aponta como principais justificativas de indicações de cesárea: desproporção cefalopélvica, cesárea prévia, parada secundária da descida e da dilatação, sofrimento fetal, macrossomia e apresentação fetal anômala ${ }^{(15)}$; já na amostra de estudo, as principais indicações foram a iteratividade (cesárea prévia) e o sofrimento fetal e a desproporção cefalopélvica foi uma justificativa pouco frequente.

Embora não tenha sido objeto deste estudo, um estudo realizado no município de São Paulo identificou que a cesárea foi mais prevalente em mulheres com idade avançada, em casos de iteratividade e idade gestacional acima de 40 semanas $^{(16)}$. Um estudo apontou ainda associação entre cesárea eletiva e: idade superior a 18 anos, ter completado pelo menos o ensino médio; possuir renda própria/trabalho remunerado; ter companheiro e realizar o pré-natal na rede suplementar ${ }^{(18)}$. Evitar a primeira cesárea é fundamental para o decréscimo a longo prazo da taxa de cesáreas, pois o parto normal após cesárea ainda é 
questionado por muitos médicos pelo risco de rotura uterina ${ }^{(17)}$.

Revisão da literatura a partir de sete ensaios clínicos que avaliaram se o manejo ativo do trabalho de parto (amniotomia artificial, uso de ocitocina endovenosa, suporte contínuo com doula e/ou enfermeiro obstetra, uso de partograma e revisão de condutas) causou impacto nas taxas de cesáreas, apontou pequena redução na taxa e não foi possível determinar o efeito de cada um dos componentes do manejo neste decréscimo. Entretanto, a satisfação foi avaliada em apenas um ensaio, e 75\% das participantes disseram estar satisfeitas com o parto, independente da via de nascimento ${ }^{(18)}$.

Em relação à satisfação com o parto, pesquisa realizada com 200 puérperas, utilizando uma escala de zero a 10, a média relacionada ao parto foi igual a nove, demonstrando alto grau de satisfação ${ }^{(19)}$. Em contraposição, percentuais relevantes de insatisfação foram encontrados em um estudo, onde $67 \%$ das nulíparas não ficaram satisfeitas e $64 \%$ atribuíram à qualidade assistencial ${ }^{(20)}$. Estudo qualitativo apontou que mulheres que tiveram como desfecho o parto normal, relataram maiores índices de satisfação, enquanto que as submetidas a cesárea, demonstravam frustração em sua fala ${ }^{(21)}$. Dentre as que se declararam satisfeitas com a cesárea, aspectos positivos ressaltados foram o medo da dor e a indicação médica para este parto durante a gestação(21).

Destaca-se que, semelhantemente aos resultados encontrados, as vivências e experiências prévias individuais das mulheres e de seus familiares foram fatores que influenciaram a mulher pela escolha do tipo de parto ${ }^{(21)}$. As mesmas identificam a influência de pessoas de seu convívio, mas ressaltam a importância de terem sido orientadas pelos profissionais durante o pré-natal e/ou trabalho de parto, e citaram em ordem de frequência: a orientação médica, do enfermeiro e da doula ${ }^{(21)}$. Entre as que não receberam informação buscaram como fonte opiniões e publicações na internet ${ }^{(21)}$ para fundamentar sua escolha.

Ante as altas taxas de cesáreas, os motivos associados à preferência e suas justificativas, torna-se necessário reavaliar a forma de organização das práticas obstétricas, com o intuito de proceder mudanças na assistência ao parto, respeitando a fisiologia da mulher e favorecer interesses de todos os envolvidos. Vale refletir que o "bom parto", seja ele vaginal ou cesárea, deve ser aquele que proporcione bem-estar materno e neonatal ${ }^{(22)}$. Decisões pelo tipo de parto e preferências das gestantes devem ser garantidas, desde que elas tenham condições de escolher, de forma isenta, aquele que melhor thes convém.

Quando avaliados os efeitos do suporte profissional na decisão pelo tipo de parto, especialmente após uma cesárea prévia, pode-se observar que mulheres que receberam o suporte apresentaram menos conflitos de decisão em relação ao planejamento e tipo de parto. Além disso, pode-se verificar maior conhecimento sobre os diferentes tipos de parto e seus resultados entre as mulheres que receberam o suporte. No entanto, de forma semelhante ao presente estudo, independente do suporte as mulheres mostraram-se satisfeitas com o parto ${ }^{(23)}$.

Vale refletir sobre a influência das parteiras tradicionais/enfermeiros obstetras na assistência ao nascimento. Um estudo de revisão, após a análise de 15 ensaios clínicos, apontou que mulheres assistidas por parteiras/enfermeiros obstetras apresentaram menores índices de analgesia/anestesia; partos vaginais 
instrumentais; amniotomia; episiotomia e nascimentos pré-termos e perdas fetais/neonatais, e, maiores índices de partos normais espontâneos. De forma que mulheres assistidas por estes profissionais tiveram redução no número de intervenções durante o trabalho de parto e parto, o que influenciou na satisfação com experiência, pois todas mostraram-se satisfeitas ${ }^{(24)}$.

Um estudo realizado com 232 mulheres em uma maternidade da Austrália teve como objetivo apresentar uma visão sobre o modelo de assistência realizado por um grupo de parteiras/obstetrizes. Como resultado observou-se que quando assistidas por estas, $87 \%$ das gestações resultou em parto vaginal, $97 \%$ de índice de satisfação com o parto e recomendariam o serviço para amigos e familiares ${ }^{(25)}$.

De forma que o acompanhamento/suporte profissional pode e deve-se constituir como uma estratégia para redução das taxas de cesáreas e complicações materno-infantis, além da melhoria dos aspectos psicológicos e emocionais das gestantes diante do processo de parto.

Salienta-se a importância do enfermeiro, em especial o enfermeiro obstetra e obstetriz no processo educativo da gestante. Este deve ser responsável pelas orientações sobre diferentes tipos de parto, sinais de alerta e de trabalho de parto (a fim de evitar internações precoces e intervenções desnecessárias) e, esclarecer dúvidas. Parte das mulheres que preferia a cesárea era por medo da dor e por acreditar na segurança do procedimento. Assim, cabe ao enfermeiro, através da educação em saúde, reduzir o 'medo' do desconhecido, promover diálogo com as mulheres, das mesmas com a equipe e com outras mulheres, auxiliando-as para que tenham escolhas e expectativas mais conscientes e, consequentemente, alcancem a satisfação com o parto. Este profissional deve estar presente nos serviços, com postura proativa e estabelecer vínculo com a clientela e demais profissionais da instituição ${ }^{(21)}$.

Os autores compreendem as limitações do presente estudo no que tange a validade externa, uma vez que os dados refletem uma realidade institucional e local e, talvez, não possam ser generalizados para outras populações e em diferentes contextos.

\section{CONCLUSÃO}

O parto cesáreo foi a via de maior prevalência, embora o parto normal tenha sido o de maior preferência pela maioria das gestantes. Primigestas e mulheres que tiveram parto normal prévio apresentaram maior desejo pelo desfecho normal. A regressão logística binária identificou que a experiência anterior influenciou na preferência pelo tipo de parto.

Não foram encontradas diferenças significativas associadas a outras variáveis, assim como não houve diferenças significativas na satisfação das puérperas, independente se o desfecho correspondeu à expectativa.

A vivência anterior do parto pode exercer influência na expectativa e escolha por determinada via de parto, porém, esta expectativa não exerceu influência na satisfação com o parto independentemente do desfecho. 


\section{Financiamento}

Fundação de Apoio à Pesquisa do Estado de Minas Gerais (FAPEMIG).

\section{REFERÊNCIAS}

1. Organização Mundial de Saúde. Declaração da OMS sobre Taxas de Cesáreas [Internet]. 2015 [acesso em: 30 ago. 2017]. Disponivel em: http://apps.who.int/iris/bitstream/10665/161442/3/WHO_RHR_15.02_por.pdf?ua=1\&ua=1.

2. Ministério da Saúde, Comissão Nacional de Incorporação de Tecnologias no SUS. Diretrizes de Atenção à Gestante: a operação Cesariana. Relatório de recomendação. Abril/2015 [Internet]. Brasília: Ministério da Saúde; 2015 [acesso em: 30 ago. 2017]. Disponível em:

http://conitec.gov.br/images/Consultas/Relatorios/2015/Relatorio_PCDTCesariana_CP.pdf.

3. Leal MC, Pereira APE, Domingues RMSM, Theme Filha MM, Dias MAB, Nakamura-Pereira $M$ et al. Intervenções obstétricas durante o trabalho de parto e parto em mulheres brasileiras de risco habitual. Cad Saude Publica [Internet]. 2014 [acesso em: 30 ago. 2017];30(Supl. 1):S17-32. Disponível em: http://dx.doi.org/10.1590/0102$311 \times 00151513$.

4. American College of Obstetricians and Gynecologists. El parto electivo antes de las 39 semanas [Internet]. 2013 [acesso em: 30 ago. 2017]. Disponível em: https://www.acog.org/Patients/Search-Patient-Education-PamphletsSpanish/Files/El-parto-electivo-antes-de-las-39-semanas.

5. Amorim MMR, Porto AMF, Souza ASR. Indicações de cesariana baseadas em evidências: parte I. Femina [Internet]. 2010 [acesso em: 30 ago. 2017];38(8):415-22. Disponível em:

http://bhpelopartonormal.pbh.gov.br/estudos_cientificos/arquivos/cesariana_baseada_evidencias_parte_l.pdf.

6. Vadeboncoeur H. Parto Natural - Mesmo após uma cesárea. São Paulo: M. Books; 2012.

7. Domingues RMSM, Dias MAB, Nakamura-Pereira M, Torres JA, d'Orsi E, Pereira APE et al. Processo de decisão pelo tipo de parto no Brasil: da preferência inicial das mulheres à via de parto final. Cad Saude Publica [Internet]. 2014 [acesso em: 30 ago. 2017];30(Supl. 1):S101-16. Disponível em: http://dx.doi.org/10.1590/0102-311X00105113.

8. Velho MB, Santos EKA, Collaço VS. Parto normal e cesárea: representações sociais de mulheres que os vivenciaram. Rev Bras Enferm [Internet]. 2014 [acesso em: 30 ago. 2017];67(2):282-9. Disponível em:

http://dx.doi.org/10.5935/0034-7167.20140038.

9. Islam M. The Safe Motherhood Initiative and beyond. Bull World Health Organ [Internet]. 2007 [acesso em: 30 ago. 2017];85(10):733-820. Disponível em: http://www.who.int/bulletin/volumes/85/10/07-045963/en/\#.

10. Vale LD, Lucena EES, Holanda CSM, Cavalvante RD, Santos MM. Preference and factors associated with the type of delivery among new mothers in a public maternity hospital. Rev Gaucha Enferm [Internet]. 2015 [acesso em: 30 ago. 2017];36(3):86-92. Disponível em: http://dx.doi.org/10.1590/1983-1447.2015.03.50032.

11. Santana FA, Lahm JV, Santos RR. Fatores que influenciam a gestante na escolha do tipo de parto. Revista da Faculdade de Ciências Médicas de Sorocaba [Internet]. 2015 [acesso em: 30 ago. 2017];17(3):123-7. Disponível em: https://revistas.pucsp.br/index.php/RFCMS/article/view/21337.

12. Freitas PF, Moreira BC, Manoel AL, Botura ACA. O parecer do Conselho Federal de Medicina, o incentivo à remuneração ao parto e as taxas de cesariana no Brasil. Cad Saude Publica [Internet]. 2015 [acesso em: 30 ago. 2017];31(9):1839-55. Disponível em: http://dx.doi.org/10.1590/0102-311X00002915.

13. Weidle WG, Medeiros CRG, Grave MTQ, Dal Bosco SM. Escolha da via de parto pela mulher: autonomia ou indução? Cad Saude Colet [Internet]. 2014 [acesso em: 30 ago. 2017];22(1):46-53. Disponível em:

http://dx.doi.org/10.1590/1414-462X201400010008.

14. Ribeiro LL, Luz GOA, Brito FEV, Penha JC, Aquino PS, Aquino CBQ. Perfil de mulheres submetidas ao parto cesariano. Revista de enfermagem UFPE on line [Internet]. 2015 [acesso em: 30 ago. 2017];9(12):1198-205. Disponível em: http://www.revista.ufpe.br/revistaenfermagem/index.php/revista/article/view/7713.

15. Liu Y, Li G, Chen Y, Wang X, Ruan Y, Zou L et al. A descriptive analysis of the indications for caesarean section in mainland China. BMC Pregnancy Childbirth [Internet]. 2014 [acesso em: 30 ago. 2017];14: 410. Disponível em:

http://doi.org/10.1186/s12884-014-0410-2. 
16. Almeida D, Cardoso AS, Rodrigues RM, Cunha A. Análise da taxa de cesarianas e das suas indicações utilizando a classificação em dez grupos. Nascer e Crescer [Internet]. 2014 [acesso em: 30 ago. 2017];23(3):134-9. Disponível em: http://www.scielo.mec.pt/scielo.php?script=sci_arttext\&pid=\$0872-07542014000500003\&lng=pt.

17. Ferrari AP, Carvalhaes MABL, Parada CMGL. Associação entre pré-natal e parto na rede de saúde suplementar e cesárea eletiva. Rev Bras Epidemiol [Internet]. 2016 [acesso em: 30 ago. 2017];19(1):75-88. Disponível em: http://dx.doi.org/10.1590/1980-5497201600010007.

18. Brown HC, Paranjothy S, Dowswell T, Thomas J. Package of care for active management in labour for reducing caesarean section rates in low-risk women. Cochrane Database Syst Rev [Internet]. 2008 [acesso em: 30 ago.

2017];(4):CD004907. Disponível em: http://dx.doi.org/10.1002/14651858.CD004907.pub3.

19. Garcia Cicuto A, Leite Belisario CR, Barco Tavares B. Puerperal women's satisfaction with their delivery. Invest Educ Enferm [Internet]. 2012 [acesso em: 30 ago. 2017];30(2):208-14. Disponível em:

http://www.scielo.org.co/scielo.php?script=sci_arttext\&pid=S0120-53072012000200005\&lng=en\&nrm=iso.

20. Benute GRG, Nomura RY, Santos AM, Zarvos MA, Lucia MCS, Francisco RPV. Preference in the process of parturition: a comparison between primiparous and nulliparous women. Rev Bras Ginecol Obstet [Internet]. 2013 [acesso em: 30 ago. 2017];35(6):281-5. Disponível em: http://dx.doi.org/10.1590/S0100-72032013000600008. 21. Nascimento RRP, Arantes SL, Souza EDC, Contrera L, Sales APA. Escolha do tipo de parto: fatores relatados por puérperas. Rev Gaucha Enferm [Internet]. 2015 [acesso em: 30 ago. 2017];36(3):86-92. Disponível em:

http://dx.doi.org/10.1590/1983-1447.2015.esp.56496.

22. Sanches NC, Mamede FV, Vivancos RBZ. Perfil das mulheres submetidas à cesareana e assistência obstétrica na maternidade pública em Ribeirão Preto. Texto contexto - enferm [Internet]. 2012 [acesso em: 30 ago.

2017];21(2):418-26. Disponível em: http://dx.doi.org/10.1590/S0104-07072012000200021.

23. Horey D, Kealy M, Davey MA, Small R, Crowther CA. Interventions for supporting pregnant women's decisionmaking about mode of birth after a caesarean. Cochrane Database Syst Rev [Internet]. 2013 [acesso em: 30 ago.

2017];(7):CD010041. Disponível em: http://dx.doi.org/10.1002/14651858.CD010041.pub2.

24. Sandall J, Soltani H, Gates S, Shennan A, Devane D. Midwife-led continuity models versus other models of care for childbearing women. Cochrane Database Syst Rev [Internet]. 2016 [acesso em: 30 ago. 2017];4:CD004667. Disponível em: http://dox.doi.org/10.1002/14651858.CD004667.pub5.

25. Lewis L, Hauck YL, Crichton C, Pemberton A, Spence M, Kelly G. An overview of the first 'no exit' midwifery group practice in a tertiary maternity hospital in Western Australia: Outcomes, satisfaction and perceptions of care. Women Birth [Internet]. 2016 [acesso em: 30 ago. 2017];29(6):494-502. Disponível em:

http://dx.doi.org/10.1016/j.wombi.2016.04.009. 